\title{
Free-breathing real-time cardiac cine MR for evaluation of left-ventricular function: Comparison to standard multi-breath-hold cardiac cine MR in 50 patients
}

\author{
Masashi Nakamura ${ }^{2 *}$, Tomoyuki Kido ${ }^{1}$, Yoshiaki Komori ${ }^{3}$, Michaela Schmidt ${ }^{5}$, Christoph Forman ${ }^{5}$, Kouki Watanabe ${ }^{4}$, \\ Teruhito Mochizuki ${ }^{1}$
}

From 19th Annual SCMR Scientific Sessions

Los Angeles, CA, USA. 27-30 January 2016

\section{Background}

Electrocardiogram (ECG)-gated breath-hold cardiac cine magnetic resonance imaging (MRI) is generally accepted as the gold standard for left-ventricular (LV) volume assessment. However, it may fail in patients with arrhythmia, impaired breath-hold capacity, and poor ECG gating. Recently, sparse real-time (RT) cine using a prototype sequence with sparse sampling and iterative reconstruction has been proposed to accelerate cine MRI (Kido et al. SCMR; 2015). The purpose of this study was to evaluate the diagnostic quality and accuracy of sparse free-breathing (FB) RT cine MRI for the quantification of LV function compared with standard multi-breath-hold cine MRI.

\section{Methods}

50 patients underwent both standard segmented cine MRI (Acc. factor 3) and sparse FB RT cine with a prototype sequence using sparse sampling and iterative reconstruction (acc. factor 12.8) on a clinical 3T MRI scanner (MAGNETOM Skyra, Siemens Healthcare, Erlangen, Germany). The cine images were obtained in a stack of 8 short-axis slices spanning the entire LV from base to apex (temporal/spatial resolution: $41 \mathrm{~ms} / 1.7 \times 1.7 \times$ $6 \mathrm{~mm}^{3}$ ). The image quality, ejection fraction (EF), enddiastolic volume (EDV), end-systolic volume (ESV), stroke volume (SV), and LV mass for sparse FB RT cine and standard cine were compared.

${ }^{2}$ Radiology, Saiseikai Matsuyama Hospital, Matsuyama, Japan

Full list of author information is available at the end of the article

\section{Results}

All sparse FB RT cine showed acceptable diagnostic image quality. Standard cine and sparse FB RT cine showed good agreement: EF $(60.3 \pm 10.3 \%$ for standard vs. $58.8 \pm 10.7 \%$ for FB RT; p = 0.09); EDV (132.5 \pm $36.7 \mathrm{ml}$ vs. $133.9 \pm 33.8 \mathrm{ml} ; \mathrm{p}=0.51)$; ESV $(54.8 \pm$ $27.4 \mathrm{ml}$ vs. $57.3 \pm 27.3 \mathrm{ml} ; \mathrm{p}=0.09)$; $\mathrm{SV}(77.6 \pm 15.9 \mathrm{ml}$ vs. $76.6 \pm 14.3 \mathrm{ml} ; \mathrm{p}=0.53)$; LV mass $(87.6 \pm 33.7 \mathrm{ml}$ vs. $81.3 \pm 31.3 \mathrm{ml} ; \mathrm{p}<0.001)$. The intra-observer and inter-observer agreement for all parameters was good.

\section{Conclusions}

Sparse FB RT cine MRI evaluates LV function with good accuracy compared with conventional multibreath-hold cine MRI. For patients with impaired breath-hold capacity, FB RT cine MRI may be clinically useful for quantitative assessment of LV function.

\section{Authors' details}

${ }^{1}$ Radiology, Ehime University, Toon, Japan. ${ }^{2}$ Radiology, Saiseikai Matsuyama Hospital, Matsuyama, Japan. ${ }^{3}$ Siemens Japan K.K., Tokyo, Japan. ${ }^{4}$ Saiseikai Matsuyama Hospital, Matsuyama, Japan. ${ }^{5}$ Siemens Healthcare GmbH, Erlangen, Germany..

Published: 27 January 2016

\section{doi:10.1186/1532-429X-18-S1-Q50}

Cite this article as: Nakamura et al:: Free-breathing real-time cardiac cine MR for evaluation of left-ventricular function: Comparison to standard multi-breath-hold cardiac cine MR in 50 patients. Journal of Cardiovascular Magnetic Resonance 2016 18(Suppl 1):Q50. 


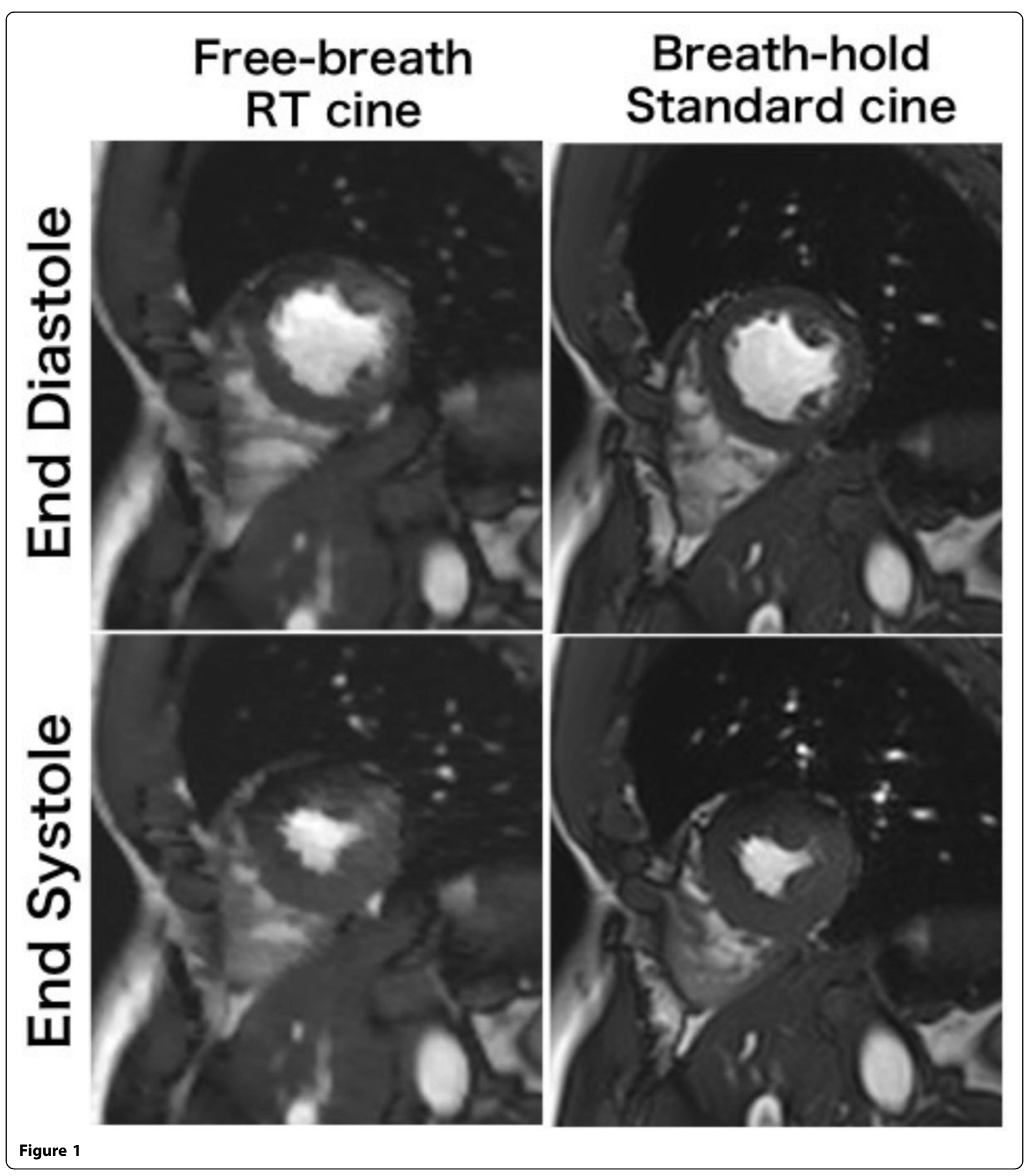

